\title{
Bilateral arm training: Why and who benefits?
}

\author{
Sandy McCombe Waller* and Jill Whitall \\ University of Maryland, School of Medicine, Department of Physical Therapy and Rehabilitation Science, \\ Baltimore, MD, USA
}

\begin{abstract}
Bilateral arm training has emerged as an approach that leads to positive outcomes in addressing upper extremity paresis after stroke. However, studies have not demonstrated improvements in all patients using current outcome measures. Furthermore, the rationale for using this type of training has been incompletely explained. The purpose of this article was to first review the theoretical justifications for the use of bilateral arm training by examining motor control and neural mechanisms underlying arm function and neural recovery, and second, to discuss examples of clinical studies using a variety of bilateral training strategies to identify who may benefit most from this approach. We argue that bilateral arm training is a necessary adjunct to unilateral training because bilateral re-training is important and best served through bilateral not unilateral training, and also, that bilateral training may help unilateral skill recovery through alternative putative mechanisms. Our review of the empirical evidence suggests that individuals at all levels of severity can benefit in some manner from bilateral training, but that not all approaches are effective for all severity levels. In addition to requesting more randomized controlled trials and studies of neurophysiological mechanisms we conclude the following: 1) Bilateral training can improve unilateral paretic limb functions of the upper extremity after stroke, however, specific training approaches need to be matched to baseline characteristics of the patients; 2) Given the importance of bilateral activities in daily life, there is a need to recognize, train and assess the important contribution of supportive role functions of the paretic arm used on its own and as part of complementary bilateral functional skills; 3) An assessment of bilateral and unilateral functioning which includes bilateral task analysis, as well as, evaluations of interlimb coordination should be included in all studies that include bilateral training; 4) Studies with thoughtful sequencing or combining of bilateral approaches or sequencing of bilateral and unilateral approaches are needed to assess if there are improved outcomes in paretic and bilateral limb function.
\end{abstract}

Keywords: Bilateral, coordination, motor control, upper extremity

\section{Introduction}

The recovery of upper extremity functional use in patients with hemiparesis has been a long standing struggle for patients and therapists alike. It has been reported that arm hemiparesis represents the dominant function limitation in as much as $80 \%$ of patients with acute stroke $[25,45]$. Intervention using traditional therapeutic approaches results in continued impairment in

*Address for correspondence: Sandy McCombe Waller PT, PhD, NCS, University of Maryland, School of Medicine, Department of Physical Therapy and Rehabilitation Science, 100 Penn Street, Baltimore, MD 21201, USA. Tel.: +1 410706 0787; Fax: +1 410706 6387; E-mail: smccombewaller@som.umaryland.edu.
$50-95 \%$ of patients, particularly after stroke $[17,18$, 37]. These stark statistics have sparked over the last 15 years an interest in empirically testing a variety of treatment strategies some old, some novel, with the hopes of achieving improved outcomes in patients with upper extremity paresis. One such approach that has been studied is bilateral arm training. In a recent metaanalysis of bilateral movement training, outcomes were positive overall during subacute and chronic phases of recovery [60]. However, studies have not shown improvements in all patients and bilateral training has not been shown to be better overall than other training approaches [50,76]. Given the heterogeneous nature of patients with hemiparesis (cortical, subcortical location, size of lesion, severity of paresis) will one ap- 
proach ever emerge as the best? Or rather, is the task at hand understanding why and with whom to use a given therapeutic approach? Based on this latter premise, the purpose of this review paper is to review the theoretical justification for the use of bilateral arm training by examining motor control and neural mechanisms underlying arm function and neural recovery, and to discuss examples of clinical studies using variations of bilateral training strategies, and their outcomes, in an effort to identify who may benefit most from this approach.

\subsection{Functional justification for bilateral arm training}

A primary reason to perform bilateral arm training is that much of what we do everyday involves the use of both arms and therefore, bilateral re-training is necessary. For example, both arms and hands are used for basic self care skills such as bathing, dressing, feeding, toileting, as well as, many other mobility functions such as carrying objects, getting up from bed or chairs, and in driving. Instrumental activities such as keyboarding, shopping and cooking also rely heavily on bilateral arm use. Moreover, older adults, who are more likely to suffer a stroke demonstrate an increase in the frequency of bilateral hand use to perform everyday activities [30]. Thus, when we consider the arm and hand deficits after stroke, we find that upper extremity paresis impacts the ability to use the affected hand in daily activities leaving many patients unable to perform bimanual tasks [8]. Interestingly, few arm training studies post stroke, regardless of the type of training, measure bilateral functional outcomes; as a result specific deficits in bilateral function are likely underreported. Of the few studies available, it is reported that bilateral finger movements needed for keyboarding show increased variability and dyscoordination [41], bilateral reaching is slower and disjointed [40,49] and bilateral arm swing is impaired [70]. In general, then, bilateral as well as unilateral re-training appears necessary for those with stroke. Therapy is often focused on training a patient to use their paretic arm in unilateral activities or, if this is not successful, to use their nonparetic arm to compensate for and accomplish tasks that were formally accomplished bilaterally. We contend that both of these practices may be less productive than re-training the two arms at the same time. To understand why we make this statement let us first consider the practice of training the paretic arm to accomplish unilateral activities.

Except for very mildly affected individuals, we argue that re-training unilateral manipulative skills should not be the only or even the primary focus particularly for those with a stroke affecting the non-dominant hand. Before suffering a stroke, individuals will use a range of unilateral skills from simple actions such as raising the arm to point or press a doorbell to complex actions such as spooning sugar into a coffee mug or writing. Typically the more complex skills are only performed by the dominant arm while the more simple skills might be performed by either arm although still the dominant arm is more likely to perform this skill and will do so with more speed and accuracy [48]. One exception to this rule of thumb is opening a door, because this, generally, depends on where the hinge is located to avoid the arm crossing the midline and impeding progression. Thus, when individuals have a stroke affecting their dominant arm, they will either wish to re-train the skills that this arm accomplished before the stroke or, if good recovery seems impossible, they will want to train their non-dominant arm to learn the complex unilateral skills, for example writing, formally executed by their dominant arm. However, for individuals with a stroke affecting their non-dominant arm, there is less re-training of most unilateral skills needed since not only were these skills fewer before the stroke but the dominant, non-paretic arm can already accomplish all of these skills. Individuals with non-dominant or those with severe dominant arm hemiparesis, then are in particular need of re-training their paretic arm to accomplish bilateral skills. What is the best method to accomplish this?

Re-training bilateral tasks is optimized by bilateral and not unilateral training. Even if the training involves a task that is part of a bilateral task, unilateral training may not lead to better bilateral function because the arms need to be coordinated. This task-specificity or transfer of learning principle is consistent with the realization that unilateral and bilateral skills have different neuromotor control mechanisms. That is, the control of each arm separately is not equivalent to the control of both together. Behaviourally, this principle is illustrated by the strong coupling mechanism that exists between the two arms when they move simultaneously. For example, spatial-temporal control of one limb alone follows Fitt's Law [12] regarding a speed/accuracy trade off, but if both hands are requested to move to two separate targets placed at different distances they will illustrate a temporal (and/or spatial) coupling that overrides the principles of Fitt's Law for each arm individually doing the same [28,29]. There are many other examples of this principle including a recent experiment by Tseng and Sholtz [68] who 
demonstrated that drawing unimanual circles uses different individual intralimb synergies than drawing circles bimanually. Countless other experiments illustrate the difficulty of combining different spatial (e.g. [14] temporal (e.g. [49] or spatio-temporal tasks (e.g. [65]) because of the tendency for coupling.

\subsection{Motor control justification for bilateral arm training}

While the so-called default coupling mechanism for symmetrical tasks is well known, it is worth taking a moment to consider other classes of bimanual skills since we began this section by suggesting that stroke patients need to re-learn bilateral functional tasks. In fact, bilateral symmetrical tasks of daily living, where temporal, spatial and force parameters of the two arms do not substantially differ and the coupling mechanism is maximally operative, are actually relatively few. Examples of these types of skills include lifting, carrying and placing a large object or two similar objects, opening and closing a two-handle drawer, pushing a cart, folding a towel and catching certain thrown objects. A second category of bilateral skills is where the two arms do the same task but in opposing or antiphase motion. Examples of these tasks are fewer but include arm swing when walking, climbing a ladder and keyboarding (although the latter is not strictly always in opposition unless one "hunts and pecks"!). These types of skills tend to be repetitive and there have been many studies that demonstrate that repetitive antiphase movements either of the whole arm [57] or of finger movements [27] are second only to inphase movements in terms of being stable movement patterns in nondisabled adults. Thus there is a rationale for thinking that these antiphase (and inphase) stable patterns which the system likes to settle into might also reflect the most easily re-activated neural pathways. On the other hand, it is less clear that the coupling advantage is utilized with these types of activities.

Finally the most common type of bilateral skills are those classed as bilateral complementary where the two arms cooperate to complete a task with each arm having a separate function that requires quite different but essentially simultaneous temporal, spatial and force parameters. Typical examples involve stabilizing an object with one hand and manipulating it with the other such as holding a jar and unscrewing the lid or holding a mug and stirring the coffee. Even tying shoelaces tends to have one hand doing more stabilization and less manipulation. Studies have consistently shown that the dominant hand is the manipulating hand while the non-dominant hand takes the stabilizing role [48]. However, studies of complementary bilateral activities in adults are very few [46]. Those bilateral activities that have been studied such as one hand pulling a drawer open while the other hand reaches into the drawer to pick out a peg $[47,77]$, or picking up a bottle, unscrewing the cap, placing it on the table, picking up a glass and pouring from the bottle into a glass [75] have demonstrated the ubiquity of a common unitary time structure for the two arms despite the varying spatial and force demands of the complementary tasks. Thus, although the bilateral tasks may have dissimilar unilateral demands, the fact remains that a strong coupling exists between the arms when they act together and this is essentially unique and different to the unilateral skills which compose the bilateral task.

Neurophysiological evidence for different control mechanisms between unilateral and bilateral versions of the same tasks comes from the fact that investigating each arm or hand separately and then combining their brain activity is not a one-to-one correspondence with the mechanisms operating in the bilateral condition. For example, in the supplementary motor area (SMA) which is strongly implicated in bimanual movements, some neurons are active during bimanual movements but not during the same movements of either arm individually $[11,26,66]$. Similarly, bimanual-specific activity has been recorded in M1 via functional magnetic resonance imaging in humans [67] and single neuron recordings in monkeys [10,11]. In a comprehensive review, Cardosa de Oliveira [1] concluded that there was ample neuroanatomical and physiological evidence to implicate separate unifying mechanisms of bilateral control that include both cortical and sub-cortical mechanisms and at least two levels of "crosstalk" between the arms (the details of which are not important for this argument and can be read in this and other reviews). Thus, from both behavioral and neurophysiological evidence, in non-disabled individuals and animals, the practice of training unilateral activities for the purpose of improving bimanual skills is not optimal. Given that individuals with stroke have a damaged central nervous system can we expect this principle to apply to them?

Individuals with stroke do show immediate coupling effects when the arms are moved together reinforcing the benefit of bilateral practice for bilateral function and suggesting that this mechanism can be exploited to produce a possible training benefit to paretic arm reaching abilities during bimanual reaching. For exam- 
ple, studies of discrete bilateral reaches [19,39,54,55] or constrained elbow flexion/extension movements [7] have all shown positive effects for various parameters of the paretic arm as this arm became more like the non-paretic arm in performance. Similarly, Rice and Newell [52] found strong coupling effects as the nonparetic arm entrained to the speed of the paretic arm during oscillating movements at preferred and fast speeds; however no benefit to the paretic arm was seen. Garry et al. [1]) also reported no facilitation effects to the impaired limb (arm or leg) during interlimb coordination conditions. It is important to recognize that it is common to see negative effects on the non-paretic arm during bilateral movements compared to unilateral movements of the same arm. These are a natural result of the coupling process since it is unlikely that the paretic arm will instantly entrain to non-paretic arm status without the reverse also occurring. Unfortunately, as mentioned earlier, the paucity of training studies that include bilateral measures as outcomes of interest means that the long-term effect of training, such bilateral symmetrical actions, is understudied and under proven. Rather, investigators have concentrated on demonstrating that bilateral training improves unilateral function which brings up the second major reason to promote bilateral arm training.

\subsection{Neurophysiological justification for bilateral arm training}

There is a neurophysiological basis for why bilateral arm training will also improve unilateral function despite our previous arguments for task-specificity in training. One mechanism through which bilateral arm training might work is through transcallosal pathways. For example, during bilateral movements, studies with transcranial magnetic stimulation show that both hemispheres have a reduction in intracortical inhibition (ICI). In contrast, increased inhibition is seen in the ipsilateral hemisphere when only one arm is activated [38,62]. McCombe Waller et al. [38] have shown that bilateral movement resulted in increased facilitation to both hemispheres and demonstrated that after short term training, bilateral training resulted in reduced ICI and increased ICF in both hemispheres, while unilateral training (dominant or nondominant) produced increased ICF and reduced ICI only the contralateral hemispheres. Training a single arm resulted in persistent inhibition and no change in facilitation in the ipsilateral hemisphere. While this study was in nondisabled individuals, the results suggest that the prac- tice of training the unilateral non-paretic arm alone for stroke patients may reinforce inhibitory processes in the lesioned hemisphere that could impede recovery of the paretic arm. Studies where inhibitory influences of the non-lesioned cortex are seen on the lesioned cortex when moving the paretic arm alone [13,44], also reinforce this point. On the other hand, bilateral training has positive neural effects for both hemispheres which may, in theory, have positive after-effects for unilateral paretic movement, as well as, the advantage of training bilateral movements.

This argument is supported by a recent study [59] where short-term bimanual training induced apparent transfer effects to subsequent unimanual performance in a subset of non-disabled subjects. Movement-related potentials (MRP) and reaction times (RT) were recorded during unimanual visuomotor trials before and after bimanual visuomotor training. Improvements in unimanual RT following the bimanual training block were associated with amplitude increases in the early MRP which, itself, is associated with movement planning. The fact that half of the subjects, who already had a low RT, did not show the same parallel findings suggests that bimanual training may not always provide a subsequent unilateral benefit particularly if unilateral performance is already optimized. Indeed, using a TMS paradigm, non-disabled subjects did not show a change of excitability of the motor cortex when both hands were voluntarily activated compared to one [51]. However, in the same study, simultaneous activation of both hands in patients with stroke caused an additional facilitation in the affected hemisphere compared to activation of the affected hand alone. We cannot comment on benefits to the movements since these were not reported. Nevertheless, taken together, the studies reported above provide some support for transcallosal facilitation or disinhibition to the lesioned hemisphere during bilateral activation or short-term training.

Long-term bilateral training studies where neurophysiological mechanisms are investigated are few. Luft et al. [34] showed an increase of activation in the contralesional hemisphere (precentral gyri and post central gyri and ipsilesional cerebellum) after 6 weeks of bilateral arm training but not after 6 weeks of unilateral arm training. This increase in contralesional activation was correlated with an increase in motor function but only in 6 out of 9 patients undergoing bilateral arm training. Two other long-term bilateral arm training studies in stroke patients that attempted to measure neurophysiological mechanisms had too few subjects to have any statistically-based conclusions $[32,64]$. The 
role of contralesional hemisphere activity, particularly in primary motor cortex is controversial [72] partly because well-recovered patients have more normal taskrelated ipsilesional activation while those with poor recovery retain contralesional activation $[69,73]$. Therefore, training methods that induce contralesional activation might be viewed as less desirable. However, there is now recognition that contralesional activation may be a necessary compensation reflecting a different strategy for movement [15] useful particularly for those who have few latent or recruitable ipsilesional pathways and little chance of good recovery [58,74]. In fact, in addition to the transcallosal mechanism already mentioned there are two other mechanisms that have been proposed for this purpose: ipsilateral uncrossed corticospinal pathways and cortical projections to bilateral brainstem pathways such as rubrospinal or propriospinal [61]. Some even argue that inducing the use of these pathways is an adaptive strategy that will facilitate motor recovery to the extent that it will enable or initiate greater recovery than would otherwise be possible in subjects with more extensive lesions [24]. Thus, activation of nonlesioned hemisphere may represent utilization of ipsilateral pathways or the induction of alternative recovery mechanism in those with fewer neural resources in the lesioned hemisphere. In this case, recovery of the paretic arm may only sustain a supportive role for the paretic arm which would argue for a greater emphasis on bilateral training on the basis of the task-specificity argument.

In comparison, it should be noted that most unilateral training paradigms are associated with reorganization in the ipsilesional cortices $[2,22,23,31,33,56]$ although at least two studies also show some contralesional activation $[31,56]$. Therefore, since subjects after unilateral training also show some recovery, one could argue that unilateral training may well be better for unilateral function. Since these studies tend to be of more mildly affected individuals, it is plausible that unilateral paretic arm training is appropriate for those with sufficient lesioned hemisphere activation as long as bilateral function is not sought. As of now, however, there has been no well-controlled study that can address whether unilateral is better than bilateral training (or vice versa) for functional recovery of the paretic limb or whether the underlying mechanisms of recovery are truly different.

In summary, there are two major arguments as to why bilateral arm training is a good idea to incorporate into stroke rehabilitation. The first argument is that individuals with stroke need to re-train bilateral skills and that this is best achieved through bilateral training given the behavioral and neurophysiological data that suggest that bilateral and unilateral skills are controlled differently. Evidence in a few studies of individuals with stroke support this observation on a short-term basis but there is little evidence from long-term training studies. Conversely, the second argument is that practicing bilateral activities may also have a positive effect on unilateral skills based on neurophysiological mechanisms that may promote at least a partial recovery. There are many studies that we can review to address the efficacy of this second argument from an empirical viewpoint and we turn now to review this body of work on bilateral training in order to examine this evidence and also to determine who benefits from this training.

\section{Review of bilateral training studies}

\subsection{Overview and categories of bilateral arm training}

In this section, we summarize categories of bilateral training, identified from the current literature, with the goal of identifying consistencies in training response relative to patient type. This exercise will help identify who benefits from bilateral training, how they benefit, and what types of training are best, given baseline subject characteristics. Studies using bilateral training have included quite varied subject pools ranging from those with dense hemiparesis to subjects with nearly full recovery of the arm but no approach has evaluated all types of subjects.

In this review we include 20 papers on bilateral training including single cohort designs, single case design experiments, and small randomized controlled trials. The method used to identify articles for review included a search of the PubMed computer data base with key words that included bilateral/bimanual control, bilateral arm training, bilateral coordination, interlimb coordination, bilateral motor control, motor control, stroke, cerebrovascular disease, arm paresis, upper extremity paresis, bilateral coupling, upper extremity control, neural control of the arms, and bilateral arm function. We also searched for references from review articles on stroke and bilateral training, bilateral training studies, behavioral and neurophysiological studies of bilateral movement and motor control. As others have noted, there is a dearth of large randomized controlled trials for bilateral arm training [71] and therefore any comparisons to other types of training are not warranted. The main purpose of this review is merely to as- 
sess whether bilateral training does provide benefits as predicted by the arguments given above, and, if so, to whom are these benefits likely to occur. Three main categories of bilateral training emerged which we categorized as 1) repetitive reaching practice with the hand fixed, 2) isolated muscle repetitive task practice, and 3) whole arm functional task training. Within these general approaches some include both bilateral and unilateral training and one, couples bilateral training with another intervention. Bilateral symmetrical activities are used in each of the training categories, at least in part. Several also included asymmetrical patterns of movement and three included complementary hand use during the training. Despite the type of bilateral training, a common feature of most training was that both hands were active and were used simultaneously. In only one study was the paretic hand totally passive.

The majority of the outcomes measured were limited to paretic limb impairments and functions with only a handful of studies evaluating bilateral task performance post training. Paretic limb impairment measures are reported in most studies. Of note, the paretic limb functional performance measures were predominantly reaching and targeting skills and/or manipulative skills where the paretic arm was tested as a primary effector. No study evaluated the performance of the paretic limb in a more supportive / assistive role, used on its own or as part of a bimanual task, except for one study which still assessed this via questionnaire and therefore not as a single outcome measure.

\subsection{Repetitive reaching with hand fixed}

Studies included in this category included training protocols in which the two hands were supported or fixed at the distal end and the arms were trained repetitively in a reaching movement. We identified two approaches, bilateral arm training with rhythmic auditory cueing (BATRAC) and robotic Mirror Image Movement Enabler (MIME). BATRAC consists of moving two unyoked handles forward and backwards in a reaching motion, completing both symmetrically (arms moving together in the same direction) and asymmetrically (one arm pushes away while the other is pulling towards). Subjects in these studies have all been chronic with mild and moderate/severe paresis. Whitall et al. 2000, evaluated BATRAC in a single cohort design with 14 subjects [76]. They showed improvements in Fugl Meyer scores, speed of arm movements, and actual use of the paretic arm predominantly in supportive roles and in bilateral tasks which were retained after 9 weeks. In a follow-up study of 22 right handed subjects, comparing outcomes for BATRAC training between those with motor dominant versus motor nondominant lesions, McCombe Waller et al., reported a differentially positive training benefit for subjects with left sided (motor dominant) stroke [42]. A small randomized controlled study of 21 subjects with mild/moderate chronic hemiparesis compared BATRAC training to dose matched unilateral therapeutic exercise [34]. In the BATRAC group, motor performance changes were seen on Fugl Meyer UE scores and the ability to lift a weight which were associated with novel nonlesioned hemisphere activations using fMRI. When analyzing reaching ability as an outcome in 18 subjects, McCombe Waller et al., demonstrated that BATRAC training improved temporal and spatial coordination that was not induced by dose matched unilateral training, indicating a task specificity training effect for bilateral training and bilateral coordination [40]. However in a study of 10 patients with stroke and 10 age matched healthy controls, subjects receiving 6 weeks of BATRAC training showed proximal and distal bilateral coordination improvements, suggesting that training patterns of bilateral coupling at one joint were transferred to other joints [41]. In contrast to the above studies and using a modified version of BATRAC (modBATRAC) - in which the training was condensed from 6 weeks to 2 weeks with longer training sessions more frequently during the week, Richards et al., found increased arm use on the Motor Activity Log but did not replicate gains in Fugl Meyer scores or speed of arm movement in patients with mild stroke severity [53]. Distribution of training and level of severity may be important factors to consider at least in terms of the original non-progressive BATRAC training.

A second approach in this category, MIME has the affected arm strapped to a forearm splint that restricts wrist and hand movement, and is connected to a robotic manipulator. When used in a bilateral training mode, the non-affected hand is placed in a similar forearm cuff. During bilateral reaching attempts the robot assists the affected hand by continuously moving the affected arm to the nonaffected arm's mirror position and orientation. Lum et al. compared MIME training (using both unimanual and bimanual modes) to a standard neurodevelopmental therapeutic training approach in 27 subjects with moderate severity paresis [35]. A treatment advantage was found for those receiving MIME with improvement in proximal movement ability and strength of the paretic arm after 1 month and in reach extent as well after 2 months of training. At a 6 month 
follow-up the two groups did not differ except that those receiving MIME had improved scores on the Functional Independence Measure. In a follow-up study Lum et al. evaluated the effectiveness of combined unilateral and bilateral MIME training to unilateral MIME and standard of care in 30 subacute patients including mild, moderate and severe paresis [36]. The combined unilateral and bilateral training resulted in similar gains to unilateral training alone; however, they report that the combined group demonstrated larger deficits at baseline testing. Gains were in Fugl Meyer UE scores, ability to more the arms out of synergy, motor power and the Functional Independence Measure. Both robot assisted training groups exceeded outcomes of the control group at post training, however by the 6 month follow-up improvements in robot and controls groups were equivalent. Lum et al suggest that robot combined therapy shows similar long term outcomes compared to conventional therapy but may accelerate the rate of recovery,

In summary, bilateral training that involves repetitive reaching with the hand fixed, showed benefits in subjects with both moderate and mild stroke severity with one study also reporting changes in neural responsiveness. In terms of paretic limb improvements, this mode of training seems to induce proximal strength gains in the paretic limb, improved ability to move the paretic arm, and increased use of the limb although that use is not always specified or measured. Increase in supportive use has been reported (qualitatively) in some of the studies although this is not accurately measured. Such a finding is consistent with the noted gains in proximal strength and control. Furthermore, one study demonstrated an increased ability to complete bilateral tasks.

\subsection{Isolated muscle repetitive tasks training}

Isolated repetitive muscle training includes bimanual training in which a single motion or muscle action is repeated as part of training. One of the most thoroughly evaluated types of training in this category are the studies conducted by Cauraugh and colleagues evaluating the effectiveness of coupling bilateral motor training with active neuromuscular stimulation of the paretic limb. In these studies, subjects generally had mild severity and met minimum motor criteria that included 10 degrees of voluntary wrist/finger extension from a flexed position. In a study with 25 subjects with chronic hemiparesis, Cauraugh et al., compared bilateral training of wrist and finger extension coupled with stimulation to unilateral paretic exercise and stimulation and a control group which moved actively [4]. Those receiving bilateral training improved better than both groups in the box and block test, the ability to sustain a muscle contraction and in reaction time of the paretic arm during both unilateral and bilateral wrist and finger movement. The unilateral exercise and stimulation also improved in all tasks compared to the control group. Further comparisons between coupled stimulation with unilateral and bilateral training in a study involving 20 subjects again, reported an added benefit to bilateral training demonstrated by higher electromyography activation levels [3]. In a follow-up study, looking at the optimal stimulation times for bilateral training, bilateral training with stimulation was better than bilateral training alone indicating that the coupling of two protocols (neuromuscular stimulation and bilateral training) was key factor to the success of this intervention [5]. In a further comparison of wrist and finger coupled stimulation with unilateral and bilateral training, Cauraugh et al., examined intralimb transfer affects in 21 patients in the chronic phase of stroke and healthy control group [6]. Paretic arm reaction time during both unilateral and bilateral reaching movements was evaluated. The coupled bilateral group showed reduced movement times overall, and increased peak velocity of the paretic arm during bilateral movements with shortened deceleration times. Unilateral training resulted in an increase in peak velocity in only unilateral paretic movements but with increase variability. While transfer was successful from distal to proximal joints, in contrast to previous work [4], a task specific training effect with bilateral versus unilateral training was evident.

A second training approach in this category includes the Bimanutrack training which consists of a robotic device that trains wrist flexion and extension or forearm pronation and supination motions in passive, active assist and resistance modes. In a single cohort study of 12 patients with chronic moderate and severe paresis, Hesse et al., evaluated the effects of adding 15 minutes of daily therapy with the arm training for 3 weeks [21]. No functional changes were evident; however improvements in impairment measures were reported. In eight of the twelve patients, tone in the wrist and fingers was decreased to a level that facilitated hygiene of the hand and reduced pain complaints. All subjects reported feeling the paretic limb was more "vivid". Effects were not retained, however, in a follow-up 3 months later. In a subsequent study, in 44 acute stroke patients with severe paresis, Hesse et al., compared bilateral train- 
ing on the Bimanutrack to electrical stimulation of the paretic wrist extensors [20]. Using the Fugl-Meyer UE test as the main outcome variable, they demonstrated that both groups increased their scores, but the bilateral robot trained group increased to a greater degree after 6 weeks of training and at the 3 month follow-up period. The authors report that the number of repetitions provided during training to the bilateral group was 10 times greater therefore it is possible the gains were due to time spent in training versus the bilateral nature of training.

Finally, Stinear and Byblow report a bilateral training approach called active-passive bilateral therapy (APBT), in which the nonparetic wrist is actively flexed and extended and drives movement of a passive paretic wrist via a manipulanda [63]. In a single cohort study of 9 acute and chronic patients with varying degrees of impairment, they used APBT in both symmetrical and asymmetrical training of six 10 min sessions per day over a 4 week time period. Five of the nine subjects improved in Fugl Meyer UE scores and these individuals also demonstrated a reduction in nonaffected wrist cortical motor maps as measured by transcranial magnetic stimulation. Two of these five subjects were acute and therefore gains due to spontaneous recovery cannot be ruled out. In those subjects with no change in movement ability no cortical map changes were found.

In summary, while gains were seen across levels of severity with this approach, the expected outcomes for isolated muscle repetitive task training seem to be tightly linked to baseline level of function. Those with moderate to severe paresis show some benefits at the impairment level and in one study with associated neural changes, however functional gains have either not been measured or not been achieved. Impressive recovery of function of the paretic arm as an effector can be induced with this training if there is preserved finger and wrist function at baseline indicating mildly impaired individuals. Limited data is available about improvements in bilateral functioning but in those with mild severity did improve reaction time of the paretic arm during bilateral movements.

\subsection{Whole arm function training}

Whole arm function training is defined as training that involved the grasp, reach and release of some object as at least some component of training. In a 12 subject single case design study, Mudie and Matyas reported gains in improved task performance of the paretic limb in block placement, peg targeting and simulat- ed drinking using the bilateral isokinietic training program (BIT) in subjects who could complete the tasks already [43]. Subjects were trained in three separate grasp and reach conditions including unilateral practice, practice with unimpaired limb guiding the impaired limb, and bilateral simultaneous movements and bilateral complementary movements. Only after the bilateral training did significant improvements in kinematic patterns of paretic limb reaching occur. Using another high functioning population of subjects, Summers et al. evaluated motor performance as well as neurophysiological response using transcranial magnetic stimulation in subjects with "most components of movement present but with impairment relative to the nonlesioned side" [64]. In this 12 subject randomized control trial, two training groups were compared with training consisting of 50 trials of a dowel placement task that was completed either simultaneously with both hands or unilaterally. Those who received bilateral training improved scores on the Motor Impairment Scale while those with unilateral training did not. Movement time decreased for the paretic limb in those who trained bilaterally, but other kinematic changes did not show any consistent training effects for either group. Subjects who participated in TMS testing, particularly for those with bilateral training, showed a decrease in nonlesioned hemisphere map volume was associated with improved motor performance of the paretic limb. Map volume changes were seen to a lesser degree in the unilateral training group and were not associated with motor performance gains. This finding, though in very few subjects, is the first to demonstrate a reduction of non-lesioned hemispheric map volume in conjunction with performance improvements after bilateral training. This reflects the studies of natural recovery mentioned in the previous section and argues against the idea that bilateral training will induce maladaptive changes.

Using a different bilateral whole arm approach, Platz et al., compared 14 "almost completely recovered inpatient hemiparetic stroke patients and 14 healthy controls training for one week using a modification of the Arm Ability Training Approach [50]. Subjects completed three training tasks including fast aiming movements, fast tapping movements, and a task to pick up and place a small wooden sticks on top of each other performed either unilaterally or bilaterally. Testing included the measurement of spatial accuracy, movement time and movement duration during a unilateral reaching task, a bilateral simultaneous reaching task and a dual task in which the paretic arm was to reach to a target and 
the nonparetic arm made a triangular finger movement The bilateral training group improved significantly in movement time for both unilateral and bilateral reaching. However, the unilateral training group also improved in both unilateral and bilateral reaching as well with no significant between group differences. It is possible that the very mild level of severity of the individuals with stroke accounted for the lack of between group differences.

Lack of difference between training groups was also demonstrated by Desrosiers et al., who compared a combination of unilateral and bilateral whole arm task training added to usual care to a control group receiving additional usual care [9]. Forty-one patients in the subacute phase, ranging from moderate to mild severity, were randomized into the two training groups both of whom improved significantly from baseline testing in arm impairment measures, arm disabilities in tasks related to activities of daily living (ADLs), and in independence in ADLs. Usual care consisted of functional activities and exercises to enhance strength, active, assisted and passive movements and sensorimotor skills of the arm as well as task training including unilateral and bilateral skills. The experimental group received systematic repetition of standardized activities related to everyday tasks involving the arms including symmetrical and asymmetrical bilateral tasks and both paretic and non paretic arm unilateral tasks which required greater mental and physical effort. Given the experimental group had many types of training it is difficult to comment on bilateral training alone. Larger and better controlled studies are needed to determine if unilateral, bilateral or combinations of training are more efficacious than current standard practice.

Contrary to the first three studies in this category, subjects with lower baseline function receiving whole arm task training have not responded as well. In a 6 subject single case design study, Lewis and Byblow compared unilateral and bilateral training of tasks that included block placement, peg targeting, peg inversion, cup inversion, rapid transfer of three cylinders and simulated drinking [32]. Additionally, using TMS they investigated neural changes in the contralateral motor pathway in the lesioned hemisphere and the ipsilateral pathways of the nonlesioned hemisphere. Subjects had baseline Fugl-Meyer UE scores ranging from $3-26$, using an abbreviated version which excluded wrist and hand functions indicating at least moderate to marked severity paresis. Furthermore, both acute and chronic patients were included. While there was a gradual improvement in task performance during the initial unilateral trials, the effect of bilateral training was not consistent between tasks within a subject or consistent across subjects. The only significant change seen was a decrease in performance during simulated drinking, rapid transfer and peg targeting. Group analyses of TMS data are not available due to the difficulty of eliciting MEPs in this group of subjects. Lewis and Byblow suggest that bilateral training may not be beneficial if the task is too complex for the patient.

In summary, there is evidence of the benefit of bilateral whole arm task training activities in improvement of 1) paretic arm function that has been associated with neural changes and 2) in speed of arm movement during both unilateral and bilateral reaching tasks, however at present; this benefit appears to be limited to those with mild paresis.

\subsection{Summary of bilateral arm training studies}

To summarize all three training categories, the use of these various bilateral training approaches have shown improvements in paretic limb mobility, paretic limb functioning, in bilateral limb function and in neural responsiveness across a range of severity levels. So the answer to the question of "who benefits" is that patients at all levels appear to benefit to some degree. However, all bilateral approaches are not effective for all severity levels. No clear pattern emerged related to chronicity of paresis and there was limited information reported regarding lesion location. Table 1 summarizes the main findings from the different types of training. Two major points arise from studying the table and the review above. First, level of impairment is a critical factor to consider when choosing a bilateral training approach. Neural resources available to a given patient may limit the type of training they can reasonably participate in and may limit neural recovery mechanisms available to them as suggested in the earlier section which in turn dictates expected outcomes. A simple example of this principle is that using the whole arm approach is not sensible for those with moderate to severe severity. A second point is that few studies assess the use of the paretic arm in a supportive role either on its own or particularly in bilateral complementary tasks. If we broadened our consideration of what is useful function of the paretic limb after stroke, we may identify a larger benefit than is currently reported especially for those with moderate severity paresis. Regaining use of the paretic limb in a supportive role used independently or as part of a bilateral task can have an impact on ability to carry out daily functions and may reduce disability. 
Table 1

Benefits of Bilateral Arm Training

\begin{tabular}{|c|c|c|c|c|c|c|}
\hline \multirow[b]{2}{*}{ Training Mode } & \multicolumn{4}{|c|}{ Paretic Benefits } & \multicolumn{2}{|c|}{ Bilateral Benefits } \\
\hline & Impairments & $\begin{array}{l}\text { Supportive } \\
\text { roles }\end{array}$ & $\begin{array}{l}\text { Manip } \\
\text { roles }\end{array}$ & $\begin{array}{c}\text { Associated } \\
\text { Neural changes }\end{array}$ & $\begin{array}{l}\text { Temp/Spatial } \\
\text { Coordination }\end{array}$ & $\begin{array}{c}\text { Bilateral } \\
\text { Tasks }\end{array}$ \\
\hline *MILD Paresis & & & & & & \\
\hline $\begin{array}{l}\text { Repetitive Reaching distal fixed } \\
\text { (Luft, et al. 2004; Lum et al. 2002, } \\
\text { 2006; McCombe Waller et al., } \\
\text { 2004,2005; Whitall et al., 2000) }\end{array}$ & $\sqrt{ }$ & $\sqrt{ }$ & $\mathrm{X}$ & $\sqrt{ }$ & $\sqrt{ }$ & $\sqrt{ }$ \\
\hline $\begin{array}{l}\text { Repetitive Isolated Motion Training } \\
\text { (Cauraugh et al, (2002, 2003, 2003, } \\
2005)\end{array}$ & $\sqrt{ }$ & Not assessed & $\sqrt{ }$ & $\sqrt{ }$ & $\sqrt{ }$ & Not assessed \\
\hline $\begin{array}{l}\text { Whole Arm Task Training } \\
\text { (Desrosiers et al. 2005; Mudie \& } \\
\text { Matyas, 2000; Platz et al., 2001, } \\
\text { Summers et al., 2007) }\end{array}$ & $\sqrt{ }$ & Not assessed & $\sqrt{ }$ & $\sqrt{ }$ & $\sqrt{ }$ & Not assessed \\
\hline $\begin{array}{l}\text { +MOD Paresis } \\
\text { Repetitive Reaching distal fixed } \\
\text { (Luft, et al. 2004; Lum et al. 2002, } \\
\text { 2006; McCombe Waller et al., 2004, } \\
\text { 2005; Whitall et al., 2000) }\end{array}$ & $\sqrt{ }$ & $\sqrt{ }$ & $\mathrm{X}$ & $\sqrt{ }$ & $\sqrt{ }$ & $\sqrt{ }$ \\
\hline $\begin{array}{l}\text { Repetitive Isolated Motion Training } \\
\text { (Stinear \& Byblow, } 2004\end{array}$ & $\sqrt{ }$ & Not assessed & Not assessed & $\sqrt{ }$ & Not assessed & Not assessed \\
\hline $\begin{array}{l}\text { Whole Arm Task Training } \\
\text { (Lewis \& Byblow, 2004) }\end{array}$ & $\mathrm{X}$ & Not assessed & $\mathrm{X}$ & $\mathrm{X}$ & Not assessed & Not assessed \\
\hline SEVERE Paresis & & & & & & \\
\hline $\begin{array}{l}\text { Repetitive Reaching distal fixed } \\
\text { (Lum, 2006) }\end{array}$ & $\sqrt{ }$ & $\mathrm{X}$ & $\mathrm{X}$ & Not assessed & $\mathrm{X}$ & $\mathrm{X}$ \\
\hline $\begin{array}{l}\text { Repetitive Isolated Motion Training } \\
\text { (Hesse et al, 2006) }\end{array}$ & $\sqrt{ }$ & Not assessed & Not assessed & Not assessed & Not assessed & Not assessed \\
\hline $\begin{array}{l}\text { Whole Arm Task Training } \\
\text { (Lewis \& Byblow, 2004) }\end{array}$ & $\mathrm{X}$ & Not assessed & $\mathrm{X}$ & $\mathrm{X}$ & Not assessed & Not assessed \\
\hline
\end{tabular}

*Mild: having preserved active wrist and finger extension movements and better

+Mod: having proximal preservation which may include preserved active wrist and finger flexion

`Severe: marked hemiparesis; $\sqrt{ }=$ improvements reported, $X=$ no improvements reported by assessed.

There is a need to develop evaluation procedures to look at improvement in supportive functions and to include the assessment of bilateral task performance in training studies to better understand the benefits that result from different modes of bilateral training.

In addition to the inclusion of large scale RCT by which the relative benefits of bilateral training and unilateral training can be assessed, other questions remain to be answered. For example, the majority of training approaches used bilateral symmetrical training. Studies are needed that examine if differential outcomes are induced by symmetrical, asymmetrical and complementary training approaches. No study discussed translation of skills learned in bilateral training to ongoing management using a home exercise program. This is an important factor that should be considered when designing training protocols which can impact the retention and progression of arm function following supervised training. Some investigators are beginning to explore the neural mechanisms that underlie the training benefit, more work is needed to better elucidate neural mechanisms associated with training and the interaction with lesion location and severity. Several studies included both unilateral and bilateral training or coupled bilateral training with other training approaches but there is no systematic attempt to determine whether this augments the response benefit for all levels of patients. How do we best combine therapeutic approaches and which approaches might work well in concert provided concurrently and or sequentially?

\section{Conclusions and suggestions}

1. Bilateral training can improve unilateral paretic limb functions of the upper extremity after stroke however; specific training approaches need to be matched to baseline characteristics of the patients.

2. Given the importance of bilateral activities in daily life, there is a need to recognize, train and as- 
sess the important contribution of supportive role functions of the paretic arm used on its own and as part of complementary bilateral functional skills. No study has assessed the impact gains in this area can have on disability of patients.

3. An assessment of bilateral and unilateral functioning which includes bilateral task analysis as well as evaluations of interlimb coordination should be included in all studies that include bilateral training.

4. Studies with thoughtful sequencing or combining of bilateral approaches or sequencing of bilateral and unilateral approaches are needed to assess if there are improved outcomes in paretic and bilateral limb function.

\section{Acknowledgements}

The authors were partially supported by the National Institute on Aging (NIA) Claude D. Pepper Older Americans Independence Center P30 AG028747.

\section{References}

[1] S. Cardosa de Oliveira, The neuronal basis of bimanual coordination: recent neurophysiological evidence and functional models, Acta Psychol (Amst) 110 (2002), 139-159.

[2] J.R. Carey, J.D. Allison and M.O. Mundale, Electromyographic study of muscular overflow during precision handgrip, Physical Therapy 63 (1983), 505-511.

[3] J.H. Cauraugh and S. Kim, Progress toward motor recovery with active neuromuscular stimulation: muscle activation pattern evidence after a stroke, J Neurol Sci 207 (2003), 25-29.

[4] J.H. Cauraugh and S. Kim, Two coupled motor recovery protocols are better than one: electromyogram-triggered neuromuscular stimulation and bilateral movements, Stroke 33 (2002), 1589-1594.

[5] J.H. Cauraugh and S.B. Kim, Chronic stroke motor recovery: duration of active neuromuscular stimulation, J Neurol Sci 215 (2003), 13-19.

[6] J.H. Cauraugh, S.B. Kim and A. Duley, Coupled bilateral movements and active neuromuscular stimulation: intralimb transfer evidence during bimanual aiming, Neurosci Lett 382 (2005), 39-44.

[7] C.L. Cunningham, M.E. Stoykov and C.B. Walter, Bilateral facilitation of motor control in chronic hemiplegia, Acta Psychol (Amst) 110 (2002), 321-337.

[8] C. Dean and F. Mackey, Motor assessment scale scores as a measure of rehabilitation outcome following stroke, Australian Journal of Physiotherapy 38 (1992), 31-35.

[9] J. Desrosiers, D. Bourbonnais, H. Corriveau, S. Gosselin and G. Bravo, Effectiveness of unilateral and symmetrical bilateral task training for arm during the subacute phase after stroke: a randomized controlled trial, Clin Rehabil 19 (2005), 581-593.
[10] O. Donchin, A. Gribova, O. Steinberg, H. Bergman, S. Cardoso de Oliveira and E. Vaadia, Local field potentials related to bimanual movements in the primary and supplementary motor cortices, Exp Brain Res 140 (2001), 46-55.

[11] O. Donchin, A. Gribova, O. Steinberg, H. Bergman and E. Vaadia, Primary motor cortex is involved in bimanual coordination, Nature 395 (1998), 274-278.

[12] P.M. Fitts, The information capacity of the human motor system in controlling the amplitude of movement, J Exp Psychol 47 (1954), 381-391.

[13] A. Floel, U. Nagorsen, K.J. Werhahn, S. Ravindran, N. Birbaumer, S. Knecht and L.G. Cohen, Influence of somatosensory input on motor function in patients with chronic stroke, Ann Neurol 56 (2004), 206-212.

[14] E.A. Franz, H.N. Zelaznik, S.S. Swinnen and C. Walter, Spatial conceptual influences on the coordination of bimanual actions: when a dual task becomes a single task, J Mot Behav 33 (2001), 103-112.

[15] W. Fries, A. Danek, K. Scheidtmann and C. Hamburger, Motor recovery following capsular stroke. Role of descending pathways from multiple motor areas, Brain 116(Pt 2) (1993), 369-382.

[16] M.I. Garry, R.E. van Steenis and J.J. Summers, Interlimb coordination following stroke, Hum Mov Sci 24 (2005), 849-864.

[17] C. Gowland, H. deBruin, J. Basmajian, N. Plews and I. Nurcea, Agonist and Antagonist Activity During Voluntary Upper Limb Movement in Patients with Stroke, Physical Therapy 72 (1992), 624-633.

[18] G.E. Gresham, T.E. Fitzpatrick, P.A. Wolf, P.M. McNamara, W.B. Kannel and T.R. Dawber, Residual Disability in Survivors of Stroke - The Framingham Study, New England Journal of Medicine 293 (1975), 954-956.

[19] M.L. Harris-Love, S. McCombe Waller and J. Whitall, Exploiting interlimb coupling to improve paretic arm reaching performance in people with chronic stroke, Arch Phys Med Rehabil 86 (2005), 2131-2137.

[20] S. Hesse, H. Schmidt and C. Werner, Machines to support motor rehabilitation after stroke: 10 years of experience in Berlin, J Rehabil Res Dev 43 (2006), 671-678, 2006.

[21] S. Hesse, C. Werner, M. Pohl, S. Rueckriem, J. Mehrholz and M.L. Lingnau, Computerized arm training improves the motor control of the severely affected arm after stroke: a singleblinded randomized trial in two centers, Stroke 36 (2005), 1960-1966.

[22] S.H. Jang, B.S. Han, Y. Chang, W.M. Byun, J. Lee and S.H. Ahn, Functional MRI evidence for motor cortex reorganization adjacent to a lesion in a primary motor cortex, Am J Phys Med Rehabil 81 (2002), 844-847.

[23] H. Johansen-Berg, H. Dawes, C. Guy, S.M. Smith, D.T. Wade and P.M. Matthews, Correlation between motor improvements and altered fMRI activity after rehabilitative therapy, Brain 125 (2002), 2731-2742.

[24] H. Johansen-Berg, M.F. Rushworth, M.D. Bogdanovic, U. Kischka, S. Wimalaratna and P.M. Matthews, The role of ipsilateral premotor cortex in hand movement after stroke, Proc Natl Acad Sci USA 99 (2002), 14518-14523.

[25] H.S. Jorgensen, H. Nakayama, H.O. Raaschou, J. Vive-Larsen, M. Stoier and T.S. Olsen, Outcome and time course of recovery in stroke. Part I: Outcome. The Copenhagen Stroke Study, Arch Phys Med Rehabil 76 (1995), 399-405.

[26] O. Kazennikov, B. Hyland, M. Corboz, A. Babalian, E.M. Rouiller and M. Wiesendanger, Neural activity of supplementary and primary motor areas in monkeys and its relation to 
bimanual and unimanual movement sequences, Neuroscience 89 (1999), 661-674.

[27] J.A. Kelso, Phase transitions and critical behavior in human bimanual coordination, Am J Physiol 246 (1984), R1000 1004.

[28] J.A. Kelso, C.A. Putnam and D. Goodman, On the space-time structure of human interlimb co-ordination, $Q J$ Exp Psychol A 35 (1983), 347-375.

[29] J.A. Kelso, D.L. Southard and D. Goodman, On the coordination of two-handed movements, J Exp Psychol Hum Percept Perform 5 (1979), 229-238.

[30] S.L. Kilbreath and R.C. Heard, Frequency of hand use in healthy older persons, Aust J Physiother 51 (2005), 119-122.

[31] C. Levy, D. Nichols, P. Schmalbrock, P. Keller and D. Chakeres, Functional MRI evidence of cortical reorganization in upper-limb stroke hemiplegia treated with contraint-induced movement therapy, Am J Phys Med Rehabil 80 (2001), 4-12.

[32] G.N. Lewis and W.D. Byblow, Neurophysiological and behavioural adaptations to a bilateral training intervention in individuals following stroke, Clin Rehabil 18 (2004), 48-59.

[33] J. Liepert, H. Bauder, W.H.R. Miltner, E. Taub and C. Weiller, Treatment-Induced Cortical Reorganization After Stroke in Humans, Stroke 31 (2000), 1210-1216.

[34] A.R. Luft, S. McCombe-Waller, J. Whitall, L.W. Forrester, R. Macko, J.D. Sorkin, J.B. Schulz, A.P. Goldberg and D.F. Hanley, Repetitive bilateral arm training and motor cortex activation in chronic stroke: a randomized controlled trial, JAMA 292 (2004), 1853-1861.

[35] P.S. Lum, C.G. Burgar, P.C. Shor, M. Majmundar and M. Van der Loos, Robot-assisted movement training compared with conventional therapy techniques for the rehabilitation of upper-limb motor function after stroke, Arch Phys Med Rehabil 83 (2002), 952-959.

[36] P.S. Lum, C.G. Burgar, M. Van der Loos, P.C. Shor, M. Majmundar and R. Yap, MIME robotic device for upper-limb neurorehabilitation in subacute stroke subjects: A follow-up study, J Rehabil Res Dev 43 (2006), 631-642.

[37] N.E. Mayo, S. Wood-Dauphinee, S. Ahmed, C. Gordon, J. Higgins, S. McEwen and N. Salbach, Disablement following stroke, Disabil Rehabil 21 (1999), 258-268.

[38] S. McCombe Waller, L.W. Forrester, F. Villagra and J. Whitall, Intracortical inhibition and facilitation with unilateral dominant, unilateral nondominant and bilateral movement tasks in left and right handed adults, Journal of Neurological Sciences.

[39] S. McCombe Waller, M. Harris-Love, W. Liu and J. Whitall, Temporal coordination of the arms during bilateral simultaneous and sequential movements in patients with chronic hemiparesis, Exp Brain Res 168 (2006), 450-454.

[40] S. McCombe Waller, W. Liu and J. Whitall, Temporal and spatial control following bimanual versus unimanual training, Human Movement Science, accepted.

[41] S. McCombe Waller and J. Whitall, Fine motor control in adults with and without chronic hemiparesis: baseline comparison to nondisabled adults and effects of bilateral arm training, Arch Phys Med Rehabil 85 (2004), 1076-1083.

[42] S. McCombe Waller and J. Whitall, Hand dominance and side of stroke affect rehabilitation in chronic stroke, Clin Rehabil 19 (2005), 544-551

[43] M.H. Mudie and T.A. Matyas, Can simultaneous bilateral movement involve the undamaged hemisphere in reconstruction of neural networks damaged by stroke? Disabil Rehabil 22 (2000), 23-37.
[44] N. Murase, J. Duque, R. Mazzocchio and L.G. Cohen, Influence of interhemispheric interactions on motor function in chronic stroke, Ann Neurol 55 (2004), 400-409.

[45] H. Nakayama, H.S. Jorgensen, H.O. Raaschou and T.S. Olsen, Recovery of Upper Extremity Function in Stroke Patients: The Copenhagen Study, Archives of Physical Medicine and Rehabilitation 75 (1994), 852-857.

[46] S.S. Obhi, Bimanual coordination: an unbalanced field of research, Motor Control 8 (2004), 111-120.

[47] S. Perrig, O. Kazennikov and M. Wiesendanger, Time structure of a goal-directed bimanual skill and its dependence on task constraints, Behav Brain Res 103 (1999), 95-104.

[48] M. Peters, Does handedness play a role in the coordination of bimanual movement? in: Interlimb Coordination: Neural Dynamical and Cognitive Constraints, S. Swinnen, H. Heuer, J. Massion and P. Casaer, eds, San Diego: Academic Press, 1994, pp. 595-617.

[49] M. Peters, Simultaneous performance of two motor activities: the factor of timing, Neuropsychologia 15 (1977), 461-465.

[50] T. Platz, S. Bock and K. Prass, Reduced skilfulness of arm motor behaviour among motor stroke patients with good clinical recovery: does it indicate reduced automaticity? Can it be improved by unilateral or bilateral training? A kinematic motion analysis study, Neuropsychologia 39 (2001), 687-698.

[51] C.I. Renner, H. Woldag, R. Atanasova and H. Hummelsheim, Change of facilitation during voluntary bilateral hand activation after stroke, J Neurol Sci 239 (2005), 25-30.

[52] M.S. Rice and K.M. Newell, Upper-extremity interlimb coupling in persons with left hemiplegia due to stroke, Arch Phys Med Rehabil 85 (2004), 629-634.

[53] L.G. Richards, C.R. Senesac, S.B. Davis, M.L. Woodbury and S.E. Nadeau, Bilateral Arm Training With Rhythmic Auditory Cueing in Chronic Stroke: Not Always Efficacious, Neurorehabil Neural Repair (2007).

[54] D.K. Rose and C.J. Winstein, Bimanual training after stroke: are two hands better than one? Top Stroke Rehabil 11 (2004), 20-30.

[55] D.K. Rose and C.J. Winstein, The co-ordination of bimanual rapid aiming movements following stroke, Clin Rehabil 19 (2005), 452-462.

[56] J.D. Schaechter, E. Kraft, T.S. Hilliard, R.M. Dijkhuizen, T. Benner, S.P. Finklestein, B.R. Rosen and S.C. Cramer, Motor recovery and cortical reorganization after constraint-induced movement therapy in stroke patients: a preliminary study, Neurorehabil Neural Repair 16 (2002), 326-338.

[57] R.C. Schmidt and M.T. Turvey, Phase-entrainment dynamics of visually coupled rhythmic movements, Biol Cybern $\mathbf{7 0}$ (1994), 369-376.

[58] D.J. Serrien, L.H. Strens, M.J. Cassidy, A.J. Thompson and P. Brown, Functional significance of the ipsilateral hemisphere during movement of the Schmidt RC and Turvey MT. Phaseentrainment dynamics of visually affected hand after stroke, Exp Neurol 190 (2004), 425-432.

[59] A.L. Smith and W.R. Staines, Cortical adaptations and motor performance improvements associated with short-term bimanual training, Brain Res 1071 (2006), 165-174.

[60] K.C. Stewart, J.H. Cauraugh and J.J. Summers, Bilateral movement training and stroke rehabilitation: a systematic review and meta-analysis, J Neurol Sci 244 (2006), 89-95.

[61] J.W. Stinear and W.D. Byblow, The contribution of cervical propriospinal premotoneurons in recovering hemiparetic stroke patients, J Clin Neurophysiol 21 (2004), 426-434. 
[62] J.W. Stinear and W.D. Byblow, Disinhibition in the human motor cortex is enhanced by synchronous upper limb movements, J Physiol 543 (2002), 307-316.

[63] J.W. Stinear and W.D. Byblow, Rhythmic bilateral movement training modulates corticomotor excitability and enhances upper limb motricity poststroke: a pilot study, J Clin Neurophysiol 21 (2004), 124-131.

[64] J.J. Summers, F.A. Kagerer, M.I. Garry, C.Y. Hiraga, A. Loftus and J.H. Cauraugh, Bilateral and unilateral movement training on upper limb function in chronic stroke patients: A TMS study, J Neurol Sci 252 (2007), 76-82.

[65] S. Swinnen, C.B. Walter and D.C. Shapiro, The coordination of limb movements with different kinematic patterns, Brain Cogn 8 (1988), 326-347.

[66] J. Tanji, K. Okano and K.C. Sato, Neuronal activity in cortical motor areas related to ipsilateral, contralateral, and bilateral digit movements of the monkey, J Neurophysiol 60 (1988), 325-343.

[67] M. Toyokura, I. Muro, T. Komiya and M. Obara, Relation of bimanual coordination to activation in the sensorimotor cortex and supplementary motor area: analysis using functional magnetic resonance imaging, Brain Res Bull 48 (1999), 211-217.

[68] Y.W. Tseng and J.P. Scholz, Unilateral vs. bilateral coordination of circle-drawing tasks, Acta Psychol (Amst) 120 (2005), 172-198.

[69] A. Turton, S. Wroe, N. Trepte, C. Fraser and R.N. Lemon, Contralateral and ipsilateral EMG responses to transcranial magnetic stimulation during recovery of arm and hand func- tion after stroke, Electroencephalogr Clin Neurophysiol 101 (1996), 316-328.

[70] K. Ustinova, J. Fung and M. Levin, Disruption of bilateral temporal coordination during arm swinging in patients with hemiparesis, Exp Brain Res 169 (2006), 194-207.

[71] R.P. Van Peppen, G. Kwakkel, S. Wood-Dauphinee, H.J. Hendriks, P.J. Van der Wees and J. Dekker, The impact of physical therapy on functional outcomes after stroke: what's the evidence? Clin Rehabil 18 (2004), 833-862.

[72] N.S. Ward, Functional reorganization of the cerebral motor system after stroke, Curr Opin Neurol 17 (2004), 725-730.

[73] N.S. Ward, M.M. Brown, A.J. Thompson and R.S. Frackowiak, Neural correlates of motor recovery after stroke: a longitudinal fMRI study, Brain 126 (2003), 2476-2496.

[74] N.S. Ward, J.M. Newton, O.B. Swayne, L. Lee, A.J. Thompson, R.J. Greenwood, J.C. Rothwell and R.S. Frackowiak, Motor system activation after subcortical stroke depends on corticospinal system integrity, Brain 129 (2006), 809-819.

[75] P.H. Weiss, M. Jeannerod, Y. Paulignan and H.J. Freund, Is the organisation of goal-directed action modality specific? A common temporal structure, Neuropsychologia 38 (2000), 1136-1147.

[76] J. Whitall, S. McCombe Waller, K.H. Silver and R.F. Macko, Repetitive bilateral arm training with rhythmic auditory cueing improves motor function in chronic hemiparetic stroke, Stroke 31 (2000), 2390-2395.

[77] M. Wiesendanger, P. Kaluzny, O. Kazennikov, A. Palmeri and S. Perrig, Temporal coordination in bimanual actions, Can J Physiol Pharmacol 72 (1994), 591-594. 